\title{
Functional Significance of Nonspatial Information in Monkey Lateral Intraparietal Area
}

\author{
Puiu F. Balan ${ }^{1}$ and Jacqueline Gottlieb ${ }^{1,2}$ \\ Departments of ${ }^{1}$ Neuroscience and ${ }^{2}$ Psychiatry, Columbia University, New York, New York 10032
}

\begin{abstract}
Although the parietal cortex is traditionally associated with spatial perception and motor planning, recent evidence shows that neurons in the lateral intraparietal area (LIP) carry both spatial and nonspatial signals. The functional significance of the nonspatial information in the parietal cortex is not understood. To address this question, we tested the effect of unilateral reversible inactivation of LIP on three behavioral tasks known to evoke nonspatial responses. Each task included a spatial component (target selection in the hemifield contralateral or ipsilateral to the inactivation) and a nonspatial component, namely limb motor planning, the estimation of elapsed time, and reward-based decisions. Although inactivation reliably impaired performance on all tasks, the deficits were spatially specific (restricted to contralateral target locations), and there were no effects on nonspatial aspects on performance. This suggests that modulatory nonspatial signals in LIP represent feedback about computations performed elsewhere rather than a primary role of LIP in these computations.
\end{abstract}

\section{Introduction}

The posterior parietal cortex has long been associated with spatial cognition: the ability to perceive, interpret, and act in the space around us. In humans, damage to the parietal lobe, in particular in the right hemisphere, produces contralesional neglect, a profound loss of awareness of the space contralateral to the lesion (Mesulam, 1999; Husain and Nachev, 2007). In the monkey, the lateral intraparietal area (LIP) has been identified as being especially important for spatial attention and saccadic eye movements (Gottlieb, 2007). LIP neurons have predominantly contralateral visual receptive fields (RFs) and selectively encode the location of objects that are intrinsically salient or that become relevant for an ongoing task (Platt and Glimcher, 1997; Gottlieb et al., 1998; Bisley and Goldberg, 2003; Balan and Gottlieb, 2006; Ipata et al., 2006; Oristaglio et al., 2006). LIP is suggested to encode a priority map: a topographic representation of the external world that guides spatial attention and oculomotor exploration (Gottlieb, 2007).

However, accumulating evidence suggests that, in addition to its spatial functions, LIP may be implicated in nonspatial computations. For example, Oristaglio et al. showed that, during a visual search task requiring a nontargeting manual report, neurons encoded the effector (limb) executing the behavioral report regardless of the location of that effector in the workspace (Oristaglio et al., 2006). Three studies show that neurons convey information about elapsed time on tasks manipulating stimulus

Received Jan. 15, 2009; revised April 22, 2009; accepted May 16, 2009.

This research was supported by National Eye Institute Grants R01 EY014697-01 and R24 EY015634 and The Keck Foundation. We thank G. Johnson and T. Hill for expert technical assistance, M. Osman and G. Asfaw for veterinary care, and the Columbia University MRI Research Center.

Correspondence should be addressed to Dr. Jacqueline Gottlieb, Department of Neuroscience, Columbia University, 1051 Riverside Drive, Kolb Research Annex, Room 569, New York, NY 10032. E-mail: jg2141@columbia.edu. D0I:10.1523/JNEUROSCI.0243-09.2009

Copyright @ 2009 Society for Neuroscience $\quad$ 0270-6474/09/298166-11\$15.00/0 duration or timing (Leon and Shadlen, 2003; Janssen and Shadlen, 2005; Maimon and Assad, 2006). Finally, neurons are sensitive to reward expectation in tasks manipulating reward probability or magnitude (Platt and Glimcher, 1999; Sugrue et al., 2004). These nonspatial responses are modulatory, in that they are strongest if neurons are activated by visual stimulation and attention in the RF and much weaker, or absent, if attention is directed elsewhere. Nevertheless, they are robust across the neural population, begging the question of whether LIP has significant contributions to nonspatial computations.

To date, this question remains open because no study has tested the extent to which experimental manipulation of LIP interferes with spatial and nonspatial function (Maunsell, 2004; Gottlieb, 2007). Therefore, we tested the effects of reversible inactivation of LIP on three tasks modeled after those used for neural recordings, which required spatial attention in conjunction with a manual report, temporal anticipation and rewardbased decisions. We used muscimol, a $\mathrm{GABA}_{\mathrm{A}}$ receptor agonist, which silences cell bodies but not fibers of passage, ensuring a localized lesion, and produces transient effects, minimizing the possibility of long-term compensation (Dias et al., 1995; Dias and Segraves, 1999). We found that inactivation produced no impairment in nonspatial aspects of performance, such as limb motor selection, temporal anticipation, and reward-based decisions, although it reliably impaired visuospatial aspects of the task (target selection and saccades in the contralateral hemifield), as expected based on previous results (Li et al., 1999; Wardak et al., 2002, 2004). We conclude that, whereas LIP receives feedback regarding nonspatial computations performed elsewhere, its primary role is in spatial and not nonspatial aspects of performance.

\section{Materials and Methods}

Two adult rhesus monkeys (Macaca mulatta) weighing 8-10 kg (one male and one female) were tested with standard behavioral and neurophysiological techniques as described previously (Balan and Gottlieb, 
2006; Oristaglio et al., 2006). All methods were approved by the Animal Care and Use Committees of Columbia University and New York State Psychiatric Institute as complying with the guidelines within the Public Health Service Guide for the Care and Use of Laboratory Animals. Data were analyzed offline using Matlab (MathWorks). Visual stimuli were displayed on an MS3400V XGA high-definition monitor $(62.5 \times 46.5 \mathrm{~cm}$ viewing area; CTX International) located $57 \mathrm{~cm}$ in front of the monkeys' eyes.

Initial mapping of LIP. Before the inactivation session, we outlined the coordinates and borders of LIP using single-neuron recordings. Recording sites were referenced to a standard grid with $1 \mathrm{~mm}$ spacing in two orthogonal directions, which was positioned in a constant orientation within the recording chamber. LIP was characterized by its location on the lateral bank of the intraparietal sulcus and its spatially tuned visual and delay period activity on the standard memory-saccade task, on which monkeys make memory saccades to briefly flashed targets (Barash et al., 1991).

Reversible inactivation. Muscimol injections were targeted to grid coordinates previously identified as yielding reliable visuospatial tuning during the recording sessions. Muscimol (Sigma) was dissolved in PBS, $\mathrm{pH} \sim 7$, to concentrations of $8.2 \pm 0.55 \mu \mathrm{g} / \mu \mathrm{l}$ (mean $\pm \mathrm{SD}$; range, $8-10$ $\mu \mathrm{g} / \mu \mathrm{l})$. Immediately before an experiment, the solution was backfilled into a $10 \mu \mathrm{l}$ Hamilton syringe (Recording Micro Syringe MRM-S02; Crist Instruments). To limit damage to neural tissue, we performed a single needle track in each experiment and infused muscimol at a single depth along the track. When possible, we confirmed the presence of spatially tuned activity before injection and the silencing of this activity after the injection by multiunit recording through an electrode attached to the Hamilton syringe. Infusion depths ranged between 2 and $6 \mathrm{~mm}$ (mean $\pm \mathrm{SD}, 3.8 \pm 1.1 \mathrm{~mm}$ ) below the cortical surface. To avoid pressure damage, injections were made in small steps of $0.5 \mu \mathrm{l}$ at $2-3 \mathrm{~min}$ intervals. The total volume injected ranged between 3 and $8.2 \mu \mathrm{l}$ (mean \pm SD, $6.09 \pm 1.53 \mu \mathrm{l})$, corresponding to a total amount of muscimol between 24 and $66 \mu \mathrm{g}$ (mean $\pm \mathrm{SD}, 50 \pm 13.3 \mu \mathrm{g})$.

A total of 12 injections were made, distributed at six sites in the right hemisphere of monkey $\mathrm{M}$ and at six sites in the left hemisphere of monkey D. After each injection, we first ran the double-saccade task as a benchmark for assessing the efficacy of the injection, followed by testing on the other tasks (in random order, counterbalanced across sessions). Behavioral testing ended within $\sim 2.5 \mathrm{~h}$ after muscimol infusion. Control data were obtained on the day after an inactivation session, using precisely the same tasks, presentation order, and parameters as during the previous session. Two injections of physiological saline injection (one in each monkey) served as an additional control for the specificity of the effects. In these sessions, we found no significant difference between control and postinjection data, showing that the effects could not be explained by nonspecific tissue damage produced by injection pressure.

Double-target saccade task. A central fixation point appeared on each trial (Fig. 1a). After a variable delay of 400-600 ms after the monkey achieved fixation, two targets were flashed successively for $100 \mathrm{~ms}$ in the contralesional and ipsilesional visual fields. The targets appeared at mirror-symmetric locations $12^{\circ}$ eccentricity along the horizontal meridian at variable delays ranging from -280 to $280 \mathrm{~ms}$ (positive numbers indicate contralesional lead). The fixation point was extinguished $100 \mathrm{~ms}$ after disappearance of the second target, and monkeys were rewarded for making a saccade to the first appearing target within $400 \mathrm{~ms}$ of fixation point offset. To avoid reinforcing a spatial bias, rewards were delivered on only $50 \%$ of correct trials, and small rewards (one-fifth of the magnitude for saccades to the first target) were also delivered for saccades to the second target. In the actual experiment, one-target trials were randomly intermixed in equal proportion with two-target trials, but the former are not analyzed for this report.

Covert visual search task. A circular array consisting of 12 figure-eight placeholders remained stably on the screen during the intertrial interval (Fig. 2a). The array appeared in a fixed configuration on each session with placeholders at $12^{\circ}$ eccentricity. Each placeholder consisted of seven equal line segments (four vertical and three horizontal) and measured $1^{\circ}$ $\times 2^{\circ}$. To begin a trial, monkeys were required to look at the central fixation point and grasp two response bars positioned at waist level, out of the monkey's view. Central fixation within a $2^{\circ}$ square window was enforced throughout the trial, and fixation break led to immediate termination of the trial without reward. After a variable fixation interval of $800-1200 \mathrm{~ms}, 50 \%$ of each of two line segments was removed from each placeholder, generating a target (an E-like shape) and 11 unique distractors. The target appeared at an unpredictable location and could face either to the right or to the left. To constrain the number of trials, only two target locations were used in each session, located at $45^{\circ}$ and $135^{\circ}$ in the contralesional and ipsilesional upper visual quadrants. Monkeys were rewarded for indicating the orientation of the target by releasing the right bar for the right-facing $\mathrm{E}$ or the left bar for the left-facing $\mathrm{E}$ within 100-1000 ms after target presentation.

Temporal anticipation task. After a $400 \mathrm{~ms}$ period of initial fixation, a visual target (a white annulus of $0.5^{\circ}$ radius) was presented. Target location was constant within a trial block, at $12^{\circ}$ eccentricity in the upper contralesional or ipsilesional hemifields $\left(45^{\circ}\right.$ or $135^{\circ}$ polar angle). At a variable delay after target onset (see Results), the fixation point was dimmed by $30 \%$ for $40 \mathrm{~ms}$, instructing the monkey to make a saccade to the target within 100-500 ms to obtain a reward.

On $91 \%$ of trials, the delay period was selected from one of two Gaussian distributions (bin size, $1 \mathrm{~ms}$ ) with means of 300 and $1600 \mathrm{~ms}$ and SDs of 50 and $70 \mathrm{~ms}$. On the remaining $9 \%$ of trials, the delay period was selected from a set of intermediate values ranging from 500 to $1400 \mathrm{~ms}$ in $1 \mathrm{~ms}$ increments. The distribution of delays was identical in control and inactivation sessions; the overall distribution in all experiments monkey $\mathrm{D}$ is shown in Figure $4 a$ (gray curve).

Free-choice saccade tasks. Two placeholders (white annuli of $0.5^{\circ}$ radius) were presented simultaneously with the fixation point. The placeholders were symmetrically located at $12^{\circ}$ eccentricity in the upper contralesional and ipsilesional hemifields $\left(45^{\circ}\right.$ and $135^{\circ}$ polar angle). In the space-reward task, after monkeys achieved central fixation, the fixation point disappeared and the two placeholders simultaneously increased in luminance (from 1.15 to $2.05 \mathrm{~cd} / \mathrm{m}^{2}$ ). Monkeys received a reward for making a saccade to either placeholder within $500 \mathrm{~ms}$ of fixation point offset. In each trial block, either the contralesional or the ipsilesional target was initially chosen to be associated with a relatively higher reward magnitude, and contingencies switched after 60-70 trials without warning. The color-reward task was similar to the space-reward task, except that reward was associated with target color and not location. After disappearance of the fixation point, one of the placeholders (randomly selected) became red and the other green. A randomly selected color was associated with a higher reward magnitude at the beginning of each block, and contingencies reversed without warning after 60-70 trials. The sum of rewards associated with both targets was kept constant throughout experiments, at $0.21 \mathrm{ml} /$ trial for the space-reward task and $0.184 \mathrm{ml} /$ trial for the color-reward task. In the space-reward task, three trial blocks were run in random order, with low and high fractional reward values of $26-74,41-59$, and $50-50 \%$. In the color-reward task, a single trial block was run with fractional rewards of 20 and $80 \%$.

Data analysis. In all experiments, the time of target presentation was measured by means of a photodiode indicating the onset of a refresh cycle, and the time of bar release was measured by means of a transistortransistor logic pulse generated whenever the monkey achieved or broke contact with the bar. Saccade onset was determined offline using acceleration and velocity criteria. Data were analyzed using nonparametric statistics (Kruskal-Wallis ANOVA, paired and nonpaired rank tests) or with two-way ANOVA to test for interaction terms as needed, unless otherwise indicated in Results. Numbers mentioned in Results represent mean and SE unless otherwise noted.

Double-target task. Error trials (in which monkeys failed to saccade to one of the two targets within the proscribed latency limits) were a small fraction of the total $(7 \%)$ and were not analyzed further. From the remaining trials, we plotted the fraction of choices of the contralesional target as a function of target onset asynchrony and used logistic regression to fit the data. The point of subjective synchrony (PSS) was defined as the point of inflection of the logistic regression curve, and its $95 \%$ confidence interval (CI) was calculated from the SE of the logistic fit.

Covert search. Error trials in which monkeys broke fixation or failed to release a bar within the proscribed latency limits represented 19\% of 
contralesional target trials and $8 \%$ of ipsilesional target trials on inactivation sessions and 10 and $8 \%$, respectively, on control sessions. These incomplete trials were not analyzed further. Response accuracy was measured as the fraction of correct choices in the set of completed trials. Reaction times (RTs) were measured for correct trials only.

Temporal anticipation. Fewer than $5-10 \%$ of trials were discarded because of fixation breaks, early or late saccades. The internal anticipation function (subjective hazard rate) representing the subjective estimation of the probability that the go signal would occur at a given point in time given that it has not occurred so far was calculated as in the study by Janssen and Shadlen (2005). First, we blurred the probability density function for delays $[f(t)$, where $t$ is the actual delay] (see Fig. $4 a$, gray line) by a normal distribution whose SD is proportional with the elapsed time $t$ :

$$
f_{S}(t)=\frac{1}{\Phi t \sqrt{2 \pi}} \int_{-\infty}^{+\infty} f(\tau) e^{-\frac{(\tau-t)^{2}}{\left(2 \Phi^{2} t^{2}\right)}} d \tau
$$

The coefficient of variation, $\Phi$, is a Weber fraction for time estimation ( $\Phi=0.26)$. Equation 1 implements the idea that the monkey's estimate of elapsed time carries uncertainty in such a way that the objective time $t_{0}$ is sensed as if occurred at $t_{0} \pm \Phi t$. The anticipation (subjective hazard rate) is then calculated using the following equation:

$$
A(t)=\frac{f_{S}(t)}{1-F S(t)} .
$$

$F S(t)$ is the cumulative distribution for $f_{\mathcal{S}}(t)$ given by the following equation:

$$
F S(t)=\int_{0}^{t} f_{S}(\tau) d \tau
$$

To analyze the data as a function of delay, saccade reaction times (SRTs) were grouped into three bins corresponding to short (170-470 ms), intermediate (500-1400 ms), and long (1430-1800 ms) delays. To analyze the data as a function of anticipation (see Fig. $6 a, b$ ), normalized individual trial SRTs were pooled across all experiments, and normalized SRTs were submitted to analysis of covariance (ANCOVA) with experimental condition (inactivation and control) as covariate. This calculates simultaneous weighted linear regression of control and inactivation data and provides information about group differences between slopes and intercepts.

Free-choice saccade tasks. Fewer than $5 \%$ of trials were discarded because of early or late saccades. Of the remaining trials, choice probability was computed over the entire session excluding the 10 trials before and after the reversal as well as the first and last 10 trials in a block. Choice data were fitted with ANCOVA, regressing the probability of choice of the contralesional target against fractional reward (the reward of the contralesional target divided by the sum of rewards for both targets). Whereas in the space-reward task we tested five values of fractional reward $(26,41,50,59$, and $74 \%)$, for the analysis in Figure $7 a$, we pooled data from the two lowest and two highest reward conditions into separate classes to increase the power of the analysis. Attempts to fit the segregated dataset with a sigmoid function resulted in a poor quality of fit that was attributable to ceiling and floor artifacts (i.e., inactivation cannot lower percentage choices below 0 , and choices in the control condition cannot exceed $100 \%$ ). Pooling the data linearized the relationship between choices and fractional reward, increased the power of the analysis, and resulted in superior fits (see $R^{2}$ values in Results). The assumption of a linear dependence on reward within a critical range is supported by previous studies of both neural activity and behavior (Platt and Glimcher, 1999; Sugrue et al., 2004; Padoa-Schioppa and Assad, 2006). a

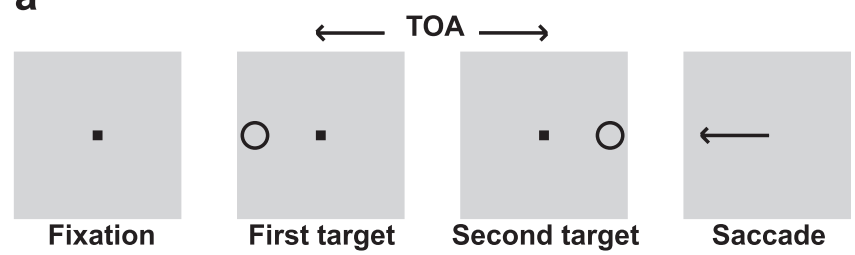

b

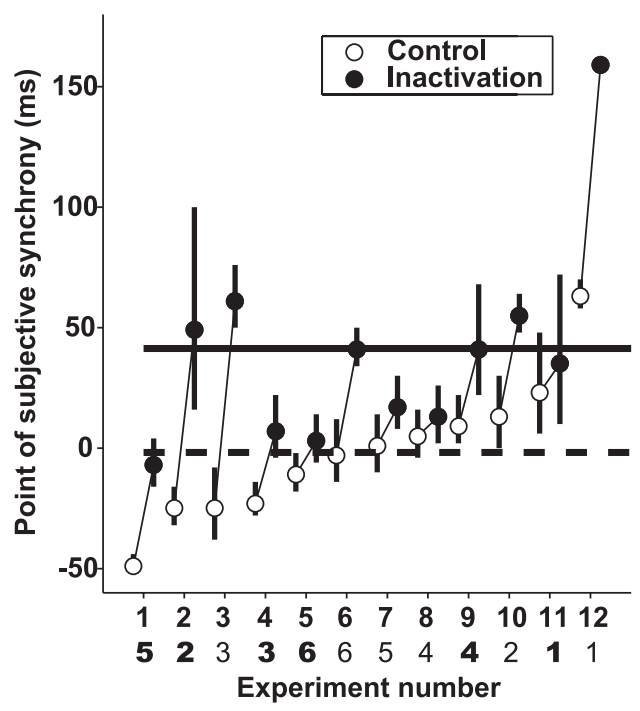

Figure 1. Double-target saccade task. $\boldsymbol{a}$, Sequence of events during the task. A trial began when the monkey achieved fixation (left). A visual target was flashed for $100 \mathrm{~ms}$ and was followed, at a variable target onset asynchrony (TOA), by a second target flashed for $100 \mathrm{~ms}$ at the diametrically opposite location. One hundred milliseconds after extinction of the second target, the fixation point was turned off and the monkeys made a saccade to the first appearing stimulus (right). $\boldsymbol{b}$, The PSS for each of the 12 experiments. Each connected pair of points shows the PSS (and the $95 \%$ Cl calculated from the SD of the logistic fit) for a pair of control (open symbols) and inactivation (filled symbols) session. Experiments are sorted in order of increasing PSS in the control session and not in the order in which they occurred. However, the bottom row of abscissa labels shows the chronological order of experiments sorted by monkey (bold symbols refer to monkey D). Average PSS for all control and inactivation sessions are indicated with the dashed and solid horizontal lines.

\section{Results}

We injected muscimol at 12 sites distributed throughout LIP in two monkeys ( ix in monkey $\mathrm{M}$ and six in monkey $\mathrm{D}$ ). There were no significant differences between monkeys, and therefore we focus on analysis of the pooled data (although individual session data are identified in some figures). Control data were obtained on the preceding or following day after recovery from the muscimol effects. Throughout, we use the term "experiment" to refer to a pair of inactivation and control sessions. We use the terms "contralesional" and "ipsilesional" to refer to locations contralateral and ipsilateral to the hemisphere that had been inactivated in the inactivation session.

\section{The double-target task}

To assess the efficacy of each inactivation, we used a benchmark double-target saccade task in which two targets were flashed in quick succession in opposite hemifields and monkeys were required to saccade to the target that had appeared first (Wardak et al., 2002) (Fig. 1a). Figure $1 b$ shows the PSS, the target onset asynchrony at which monkeys did not reliably judge which target appeared first, in each of the 12 paired inactivation and control 


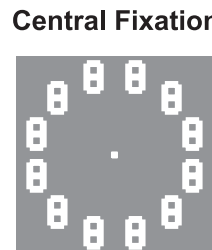

Search ON

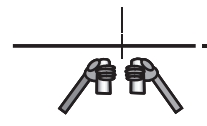

b

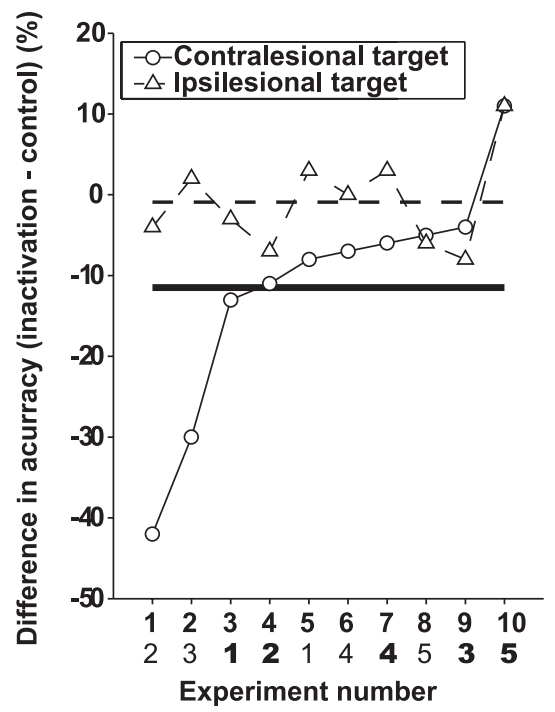

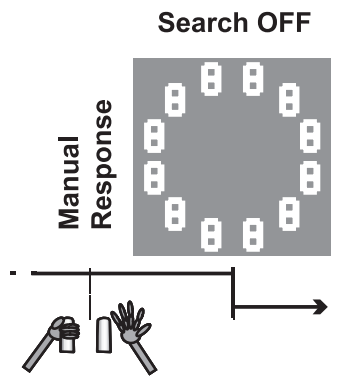

C

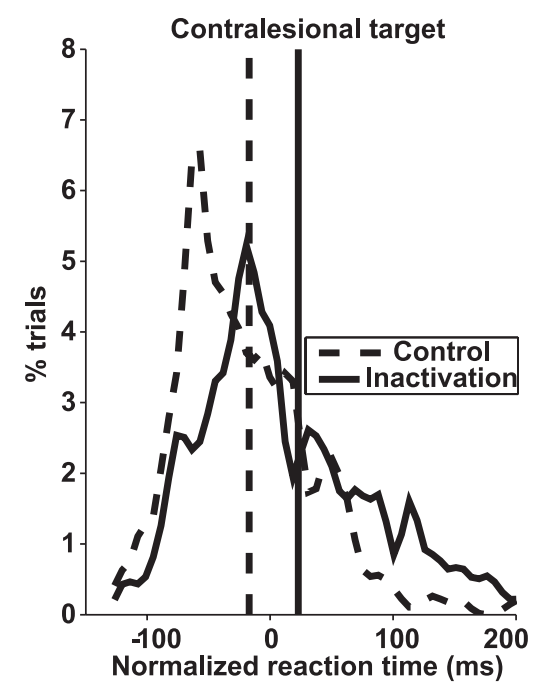

Figure 2. Covert visual search task. $\boldsymbol{a}$, Sequence of events during a representative trial. A stable placeholder array remained visible throughout the intertrial interval. To initiate a trial, monkeys were required to achieve central fixation and grasp two response bars (left). The search display was then presented by removing a fraction of two lines from each display element (middle). This revealed a display with a target (a right- or left-facing letter E) and 11 unique distractors. Monkeys were rewarded for indicating the orientation of the target by releasing grasp of a bar held in the right or left paw, without shifting gaze from the center (Manual Response). The trial ended with removal of the fixation point, a reward, if appropriate, and restoration of the placeholder display $300 \mathrm{~ms}$ after the bar release. $\boldsymbol{b}$, Inactivation effects on response accuracy in each experiment. Each point shows the difference in percentage correct between an inactivation and control session, when the target was contralesional (circles) or ipsilesional (triangles) to the inactivated hemisphere. Experiments are arranged in order of increasing effect for the contralesional target, and chronological order is given in the bottom row of abscissa labels (bold symbols refer to monkey D). The solid and dashed horizontal lines show the average difference for contralesional and ipsilesional targets, respectively. c, Distribution of normalized reaction times, pooled across all control (dashed) and inactivation (solid) sessions for contralesional targets. Reaction times were normalized by subtracting the average reaction time in each experiment (each pair of control-inactivation session). Vertical lines show averages for control and inactivation data.

sessions. For clarity of presentation, the data are sorted in order of PSS in the control condition; the bottom row of labels shows the chronological order of sessions sorted by monkey. In control sessions, the average PSS was close to 0 (dashed line) $(-1.8 \pm 8.6$ ms; $p=0.83$ relative to 0 ). This shows that monkeys achieved good temporal discrimination without a consistent spatial bias and that performance recovered fully on control sessions, i.e., there were no residual effects of muscimol across days. However, after inactivation, there was a significant increase in the average PSS (solid line) ( $41.3 \pm 13.1 \mathrm{~ms} ; p<0.012$, paired signed rank test between inactivation and control PSS), indicating a reliable ipsilesional bias: the contralesional target had to lead by $\sim 41 \mathrm{~ms}$ for the monkeys to judge it as appearing simultaneously with the ipsilesional one. An ipsilesional bias was seen in all 12 experiments, and differences between the PSS reached statistical significance in 9 of 12 cases ( $p<0.05$ based on CI). These findings establish the effectiveness of the inactivation and reproduce the spatial bias reported previously (Wardak et al., 2002).
One possible concern is that repeated inactivations produced long-term mechanical damage that may nonspecifically affect fibers of passage. However, if that were the case, we should find a gradual increase in PSS in control sessions over the course of experiments, indicating a persistent, non-recovering deficit; no such effect was found (Fig. $1 b$, control data; linear regression of control PSS as a function of experiment order, $p>0.1$ in each monkey). A second concern was that some injections were insufficiently large to produce an effect. To evaluate this, we calculated correlations between the inactivation effect and injection volume and muscimol amount across the entire dataset. Neither correlation was statistically significant (volume, $r=-0.050, p=0.10$; amount, $r=-0.047, p=0.13$ ), suggesting that the amount of injected muscimol was not a limiting factor in the results.

\section{Visuospatial but not manual deficits during covert search}

We recently found that, during a covert visual search task, the planning of a nontargeting manual report (release of a grasp with the right or left hand) elicited strong limb-specific responses in LIP (Oristaglio et al., 2006). Therefore, to see whether suggesting LIP has heretofore unrecognized contributions to skeletomotor planning, we examined the effect of inactivation on a task similar to that used by Oristaglio et al. (Fig. 2a). While maintaining central fixation, monkeys had to find a peripheral visual target (an E-like shape) presented among distractors and report target orientation by releasing a bar grasped in the right or left paw (Fig. 2a). Task performance declines as a function of the number of distractors, indicating that the task was effortful and attentionally demanding (Balan et al., 2008). Critically, the task dissociates visual attention (linked to the visual display) from limb motor planning (linked to the right of left limb), allowing us to distinguish between inactivation effects on each function.

Inactivation produced a consistent decline in accuracy and increase in RT if the target appeared in the contralesional hemifield. For contralesional targets, accuracy declined in 9 of 10 experiments, with an average decrease of $-11.5 \pm 4.9 \%$ (Fig. $2 b$, circles) ( $p<0.04$ relative to $0 ; p<0.05$, paired-rank test between inactivation and control). For ipsilesional targets, accuracy was not affected [Fig. $2 b$, triangles; average change of $-0.9 \pm 2.0 \%$ (dashed line), $p=0.64$ relative to 0 ]. Inactivation also produced a significant increase in RTs for contralesional targets in 8 of 10 individual sessions and across the dataset (Fig. 2c) (average RT, normalized by subtracting the experiment mean, was $22.9 \pm 5.9$ $\mathrm{ms}$ in inactivation sessions vs $-17.1 \pm 3.6 \mathrm{~ms}$ in control sessions; $\left.p<10^{-8}\right)$. Inactivation did not affect RT for ipsilesional targets $(5.2 \pm 3.7 \mathrm{~ms}$ after inactivation vs $-4.9 \pm 3.8$ in control sessions; $p=0.058$; significant increase in 2 of 10 sessions). Thus, inacti- 
vation produced a visuospatial deficit specific to the contralesional hemifield.

The fact that deficits were restricted to contralesional target locations rules out the possibility that inactivation globally impaired manual performance (i.e., a bimanual deficit). However, inactivation may have produced more subtle deficits for release of the contralateral or ipsilateral limbs or for a specific combination of target location or limb. To evaluate this possibility, we further subdivided the data for each target location according to the active limb (Fig. 3). When the target was contralesional, inactivation had highly significant effects on both accuracy (Fig. $3 a$ ) and RT (Fig. 3b), but there was no significant effect of limb or interaction between limb and inactivation (for accuracy, $p<0.0005$ for inactivation, $p=1.0$ for limb, and $p=$ 0.85 for interaction, two-way ANOVA; for RT, $p<10^{-9}, p>0.1$, and $p>0.4$ ). For ipsilesional targets (middle panel) there was no overall effect of inactivation, nor an effect of limb or interaction between inactivation and limb in either accuracy or RT (all $p>0.2$ ). The main effect of inactivation remained significant if data were pooled across target locations (right) $(p<$ 0.02 for both accuracy and RT), but, again, there was no effect of limb or interaction between limb and inactivation (all $p>$ $0.5)$. These findings were replicated in each of the 10 individual experiments. Thus, the magnitude of the behavioral deficit was determined solely by the location of the target regardless of whether monkeys released the right or left limb.

We performed a number of additional analyses to validate these findings. To rule out the possibility that repeated injections caused permanent mechanical damage, we computed a lateralization index, measuring the relative performance in the contralesional and ipsilesional field in control sessions. The indices (calculated as the difference in performance accuracy or RT between contralesional and ipsilesional target locations, normalized by the sum) were not significantly different from 0 for either monkey (all $p>0.2$, indicating no significant lateralization), and there was no trend as a function of session order (one-way ANOVA, all $p>0.2$ for effect of session). Thus, control performance was balanced in the contralesional and ipsilesional field, and there was no evidence for an increasing ipsilesional bias indicative of longterm damage. Second, we considered the possibility that the lack of limb-specific effects was attributable to insufficient injection volume in some cases. To evaluate this possibility, we calculated correlation coefficients between the volume and quantity of injected muscimol and the inactivation effect (the normalized difreaction times. the same format as in $\boldsymbol{b}$.
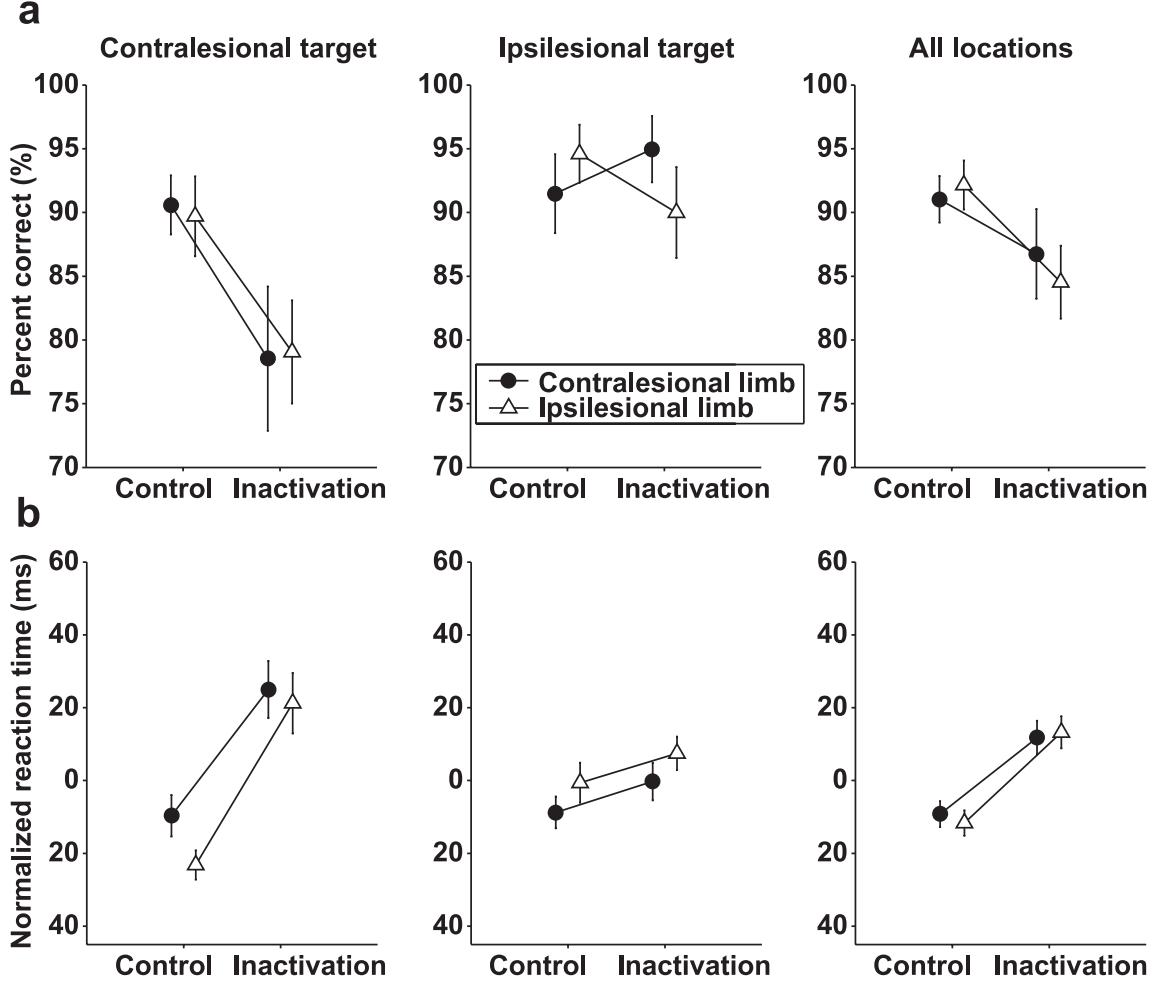

Figure 3. Covert visual search task: effect of target location and limb. $\boldsymbol{a}$, Average accuracy in trials sorted according to target location, active limb, and inactivation condition. Each point represents the mean and SE across sessions. $\boldsymbol{b}$, Same as in $\boldsymbol{a}$ but for
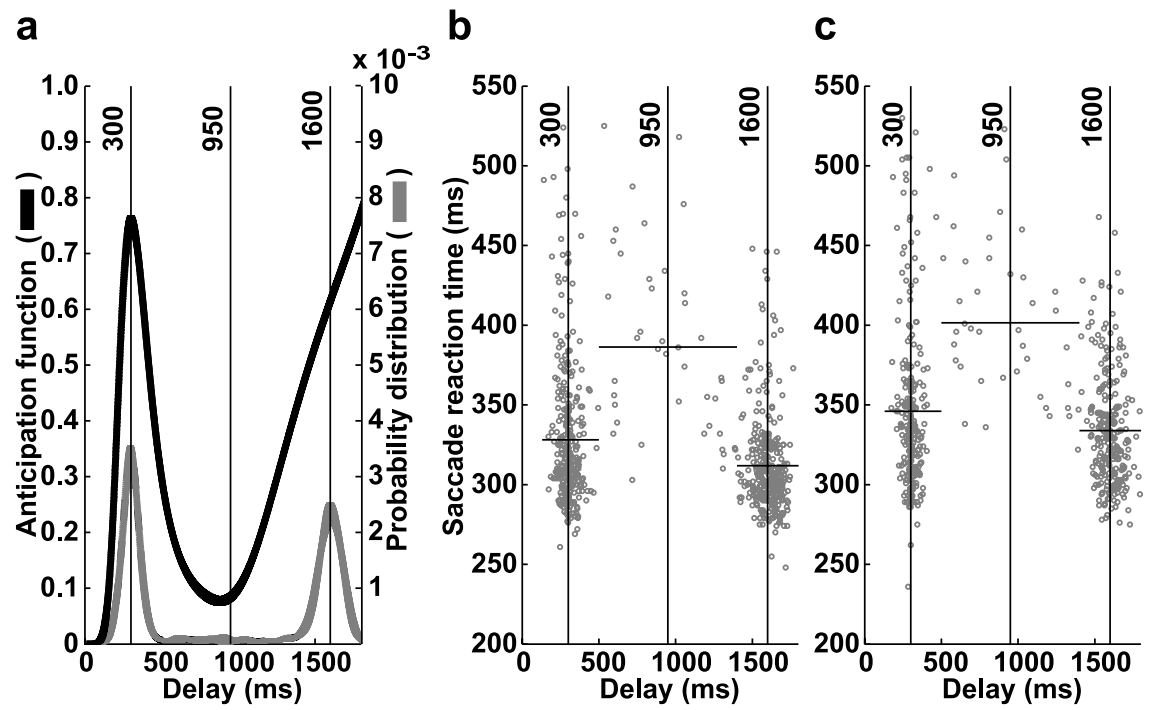

Figure 4. Temporal anticipation task. $\boldsymbol{a}$, The gray curve shows the probability distribution of the delay values used (pooled across all experiments in monkey D; bin size, $1 \mathrm{~ms}$ ). The solid curve shows the subjective anticipation (hazard) function calculated from the delay distribution, as described in Materials and Methods. Shading shows \pm 1 SD. $\boldsymbol{b}$, Saccade reaction times for contralesional (rightward) saccades in monkey D, collapsed across all control sessions. Each point represents one trial. The vertical lines show the average delay in each delay group. The short horizontal lines show the average reaction time for each delay category; their horizontal extent indicates the range of delays included in each category (short delays, $170-470 \mathrm{~ms}$; intermediate delays, $500-1400 \mathrm{~ms}$; and long delays, $1430-1800 \mathrm{~ms}$ ). c, Results from inactivation sessions with contralesional targets in monkey D, in

ference between accuracy and RT in inactivation and control sessions). This resulted in 24 correlation coefficients [ 6 combinations of target location and limb $\times 2$ performance parameters (accuracy and reaction time) $\times 2$ injection parameters (injected volume and quantity)], of which none were statistically signifi- 
cant (all $p>0.3)$. Similarly, there was no correlation between the magnitude of spatial and nonspatial deficits (all $p>0.3$ ). Finally, there was no dependence of the effects on the order of the experiment (linear regression, $p>0.1$ ) showing that the effects remained stable throughout the study. Together, these findings rule out artifacts related to the injection procedures that may affect the results.

\section{Temporal anticipation}

A previous study has shown that LIP neurons are modulated by an estimation of elapsed time in a task that varies the motor preparatory period before a saccade (Janssen and Shadlen, 2005). To evaluate the potential role of LIP in the computation of elapsed time, we tested the effect of muscimol inactivation in a task similar to that used by Janssen and Shadlen. Monkeys made a simple visually guided saccade to an isolated, clearly visible target, and the length of the preparatory period, between achievement of central fixation and the saccade go signal, was systematically varied. Delay length on each trial was randomly selected from an underlying bimodal distribution (Fig. $4 a$, gray curve).

Confirming the results of Janssen and Shadlen, SRTs were sensitive to the length of the preparatory period. Figure $4 b$ illustrates this sensitivity for contralesional target trials in control sessions from monkey D. SRTs were highest for intermediate delays when the go signal was very unlikely to occur (386 $\pm 9 \mathrm{~ms})$ but were lower for short delays $(328 \pm 2 \mathrm{~ms})$ and lower still for the longest delays, when the monkey could be virtually certain that the go signal was imminent $(312 \pm 2 \mathrm{~ms} ; p=0$ for effect of delay and $p<0.01$ for each pairwise difference, one-way ANOVA). Inactivation (Fig. $4 c$ ) produced a global elevation of SRT, consistent with a contralesional deficit in saccade target selection and/or motor planning (average SRT of $345 \pm 2 \mathrm{~ms}$ after inactivation vs $324 \pm 2 \mathrm{~ms}$ for control sessions; $p=0$ ). However, inactivation did not abolish the dependence of SRT on delay. The main effect of delay remained highly significant after inactivation (one-way ANOVA, $p=0 ; p<0.01$ for each pairwise comparison), and a two-way ANOVA combining control and inactivation data revealed a highly significant main effect of delay $(p=0)$, a significant effect of inactivation $\left(p<10^{-6}\right)$, but no interaction between delay and inactivation $(p=0.65)$. Thus, inactivation did not affect the dependence of SRT on delay.

This pattern was seen in the entire dataset (Fig. 5). For contralesional targets (Fig. 5a), average SRTs (black symbols) showed highly significant main effects of delay and inactivation (two-way ANOVA, $p=0$ for each main effect) but no interaction between the two ( $p=0.13$; SRTs for intermediate, early, and late delays were $379 \pm 9.9,319 \pm 5.1$, and $300 \pm 4.8 \mathrm{~ms}$ for control and $395 \pm 6.4,348 \pm 7.4$, and $326 \pm 6.5 \mathrm{~ms}$ for inactivation sessions). For ipsilesional targets (Fig. $5 b$ ), there was a significant main effect of delay $(p=0)$ but no effect of inactivation $(p=$ 0.17 ) and no interaction between inactivation and delay ( $p=$ 0.93; average SRTs were, in control sessions, $374 \pm 7.3,334 \pm 5.9$, and $308 \pm 2.8 \mathrm{~ms}$, and, in inactivation sessions, $377 \pm 6.7,337 \pm$ 5.6 , and $309 \pm 4.5 \mathrm{~ms}$ ). To examine the consistency of this pattern, we performed the analysis in individual experiments (denoted by gray symbols; six from monkey D, five from monkey $\mathrm{M})$. A significant main effect of delay length was found in 11 of 11 experiments for both contralesional targets and ipsilesional targets, in control and inactivation sessions (two-way ANOVA, all $p<0.01)$. Significant elevations of RT after inactivation were found in 7 of 11 sessions for contralesional targets $(p<0.01)$ but in only 2 of 11 sessions for ipsilesional targets. In no experiment was there a significant interaction between inactivation effects

\section{a Contralesional targets}

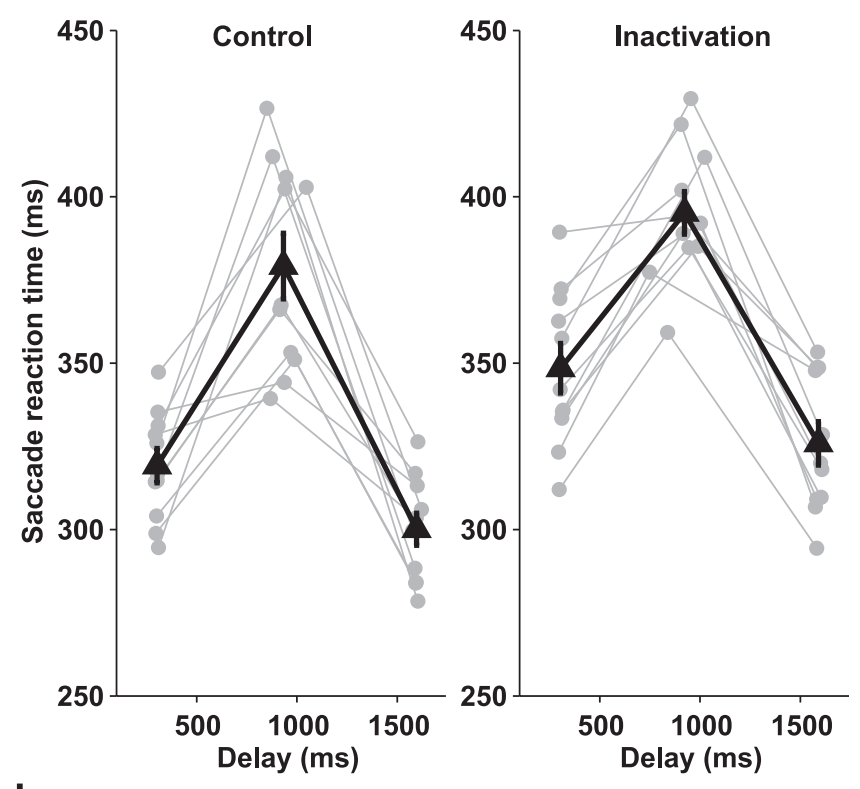

b Ipsilesional targets
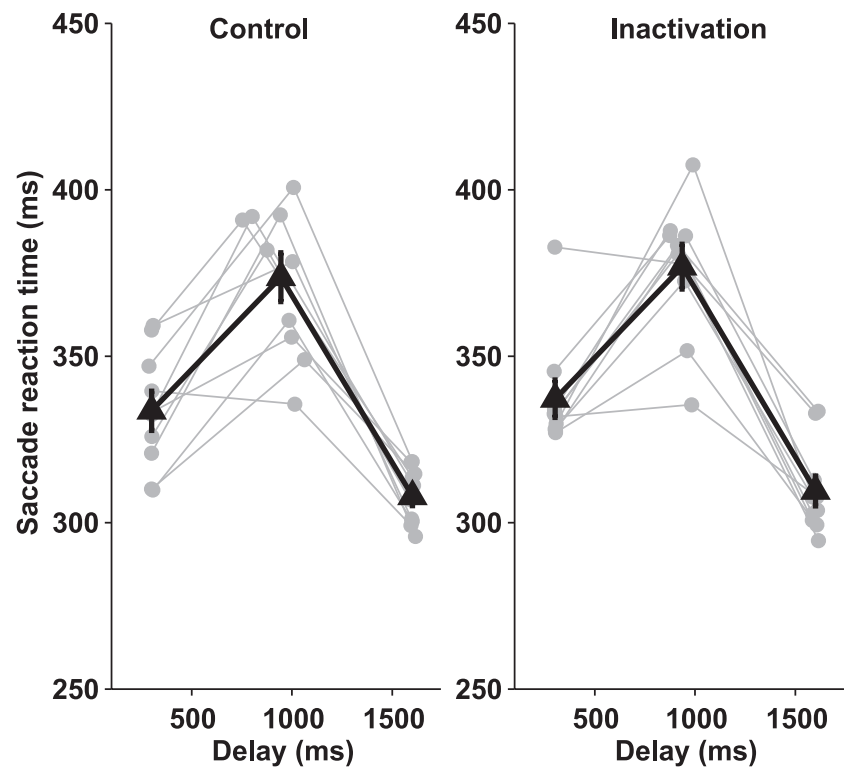

Figure 5. Temporal anticipation task: dependence of SRT on delay an SRT as a function of delay on contralesional target trials, in control (left) and inactivation (right) sessions. Each triplet of connected gray symbols shows the average reaction time in the three delay bins, for one session. The filled triangles show the mean and SEs across all sessions. $\boldsymbol{b}$, Same as in $\boldsymbol{a}$, for ipsilesional saccades.

and delay (all $p>0.2$ ). We finally examined whether the inactivation affected the second moment of the SRT distribution, the variance. In control sessions, variance was largest for intermediate delays $\left(p<10^{-7}\right.$ for effect of delay for both contralesional and ipsilesional targets; two-way ANOVA), showing that it was also sensitive to temporal anticipation. However, this relationship remained entirely unaffected by inactivation for either target location (contralesional targets, $p=0.86$ and 0.11 for main effect of inactivation and interaction with delay, respectively; ipsilesional targets, $p=0.66$ and 0.36$)$.

Janssen and Shadlen (2005) argued that LIP encodes an internal hazard function: the estimated likelihood that a go signal would occur at a given point in time given that it has not occurred 
so far. The anticipation function (see Materials and Methods) is a highly nonlinear function of delay length that peaks at the first mode of the underlying distribution, declines sharply for the infrequent intermediate delays, and increases again for long delays (Fig. 4a, black trace). Thus, we performed a second analysis of SRT as a function of anticipation rather than delay length.

As shown in Figure 6, $a$ and $b$, the relationship between SRT and anticipation was well captured by a linear regression. Regressions were highly significant in each case (contralesional control, $R^{2}=0.46$, $p<10^{-15}$; contralesional inactivation, $R^{2}=0.64, p=0$; ipsilesional control, $R^{2}=0.47, p<10^{-12}$; ipsilesional inactivation, $R^{2}=0.40, p<10^{-11}$ ) and were comparable in quality with exponential and polynomial fits of the data. The square root of the normalized sum-square-errors for the linear, exponential, and polynomial fits were 7.0, 7.0, and 6.0 for control and $7.0,7.0$, and 7.0 for inactivation data (contralesional targets), and 8.0, 7.0, and 7.0 for control and 8.0, 8.0, and 8.0 for inactivation data (ipsilesional targets). By definition, the independent variable is nonhomogeneously distributed (trials with high anticipation were much more frequent than those with low anticipation); to circumvent this, we used weighted regression, which compensates for the difference in the variance of the estimate for high and low anticipation values. Analysis of the residuals showed that they were normally distributed and independent of anticipation, further validating the linear model.

To estimate whether inactivation affected temporal anticipation, we simultaneously fit control and inactivation data using ANCOVA. In this analysis, the dependence of SRT on anticipation is captured by the slope of the fitted function, whereas changes in average SRT are reflected in the intercept. For contralesional targets (Fig. $6 a$, left and middle), the best fits had identical slopes for inactivation and control data $(-122 \mathrm{~ms} /$ anticipation unit) but a significantly higher intercept for inactivation relative to control SRT (normalized SRT of $91 \mathrm{~ms}$ vs $63 \mathrm{~ms} ; p=0$ ). Thus, inactivation produced a global elevation of SRT but no change in the sensitivity to anticipation. For ipsilesional targets (Fig. $6 b$, left and middle), the best fit produced identical slopes $(-89$ ms/anticipation unit) and equivalent intercepts ( $53 \mathrm{~ms}$ vs 55 $\mathrm{ms} ; p=0.21$ ), showing that inactivation had no significant effect on either global SRT or its sensitivity to elapsed time. As a final verification, we binned the data into 10 anticipation bins and calculated the difference between control and inactivation SRT in each bin (Fig. $6 a, b$, right panels). The effect of inactivation was only significant for contralesional targets and was entirely unrelated to anticipation (linear regression, $p=$ ipsilesional targets.
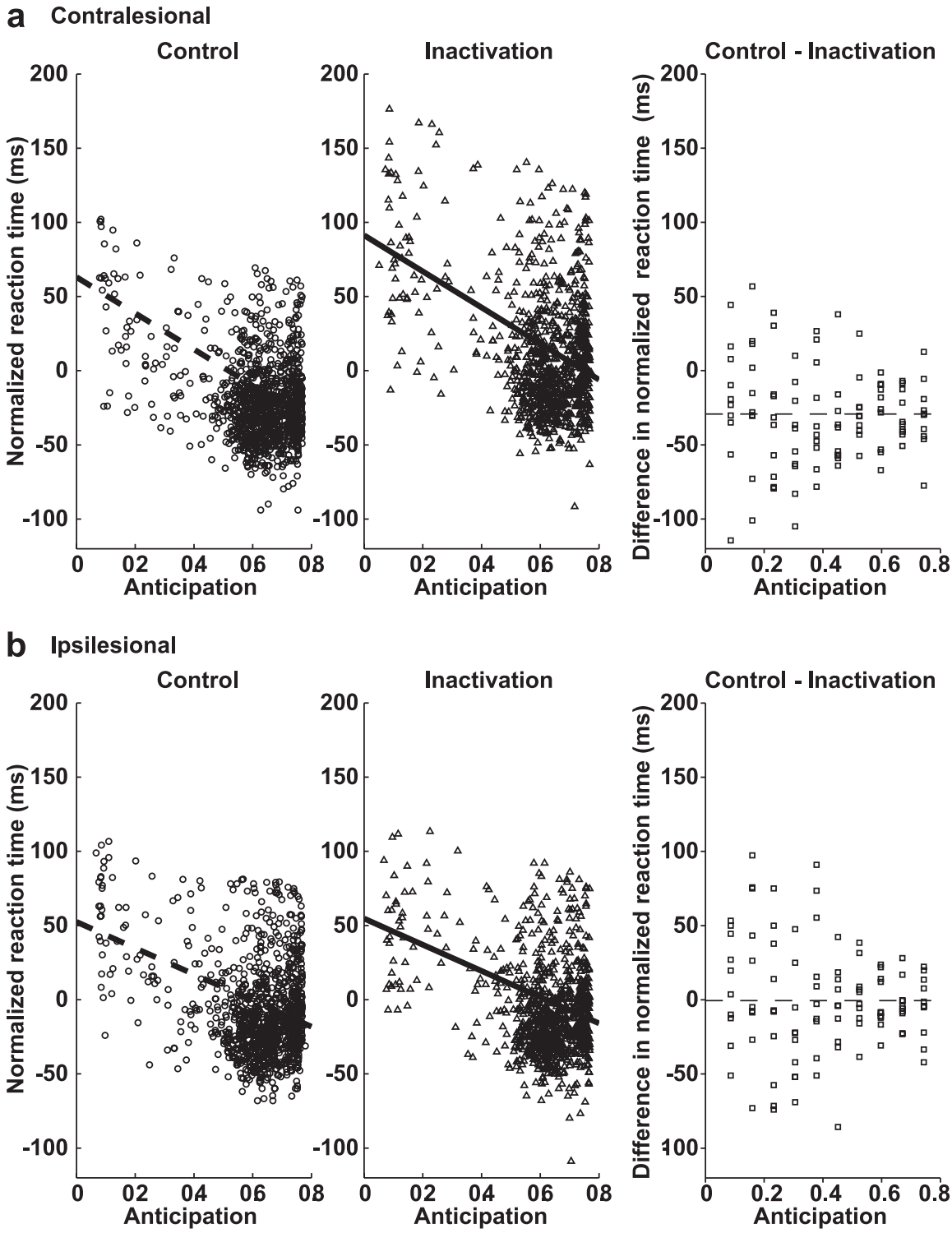

Figure 6. Temporal anticipation task: dependence of SRT on anticipation. $\boldsymbol{a}$, Normalized SRT as a function of anticipation for contralesional targets in control (left) and inactivation (right) sessions. Each point shows an individual trial. The lines show the right shows the difference between control and inactivation SRT in 10 equal anticipation bins; each point shows the difference in one pair of control and inactivation session. The dashed line shows the best-fit weighted linear regression. $\boldsymbol{b}$, Same as $\boldsymbol{a}$, for

0.8 for contralesional and $p=0.9$ for ipsilesional targets). In summary, analysis of the data as a function of anticipation corroborates the conclusions of the delay-based analysis: inactivation elevated SRTs for contralesional targets but did not affect the monkeys' sensitivity to temporal task parameters.

We performed a number of additional analyses to validate our methods. In control sessions, SRTs were equivalent for contralesional and ipsilesional targets, showing that performance was even across the tested locations; the differences between the hemifields remained stable across sessions (one-way ANOVA, $p=0.75$ for effect of session), providing no evidence for a longterm contralesional deficit. To test for a possible effect of injection size, we calculated the correlations between magnitude of inactivation effect and the amount and volume of injected muscimol. The four correlation coefficients $(2$ target locations $\times 2$ injection parameters) were not significant $(p>0.24$ in each 
a
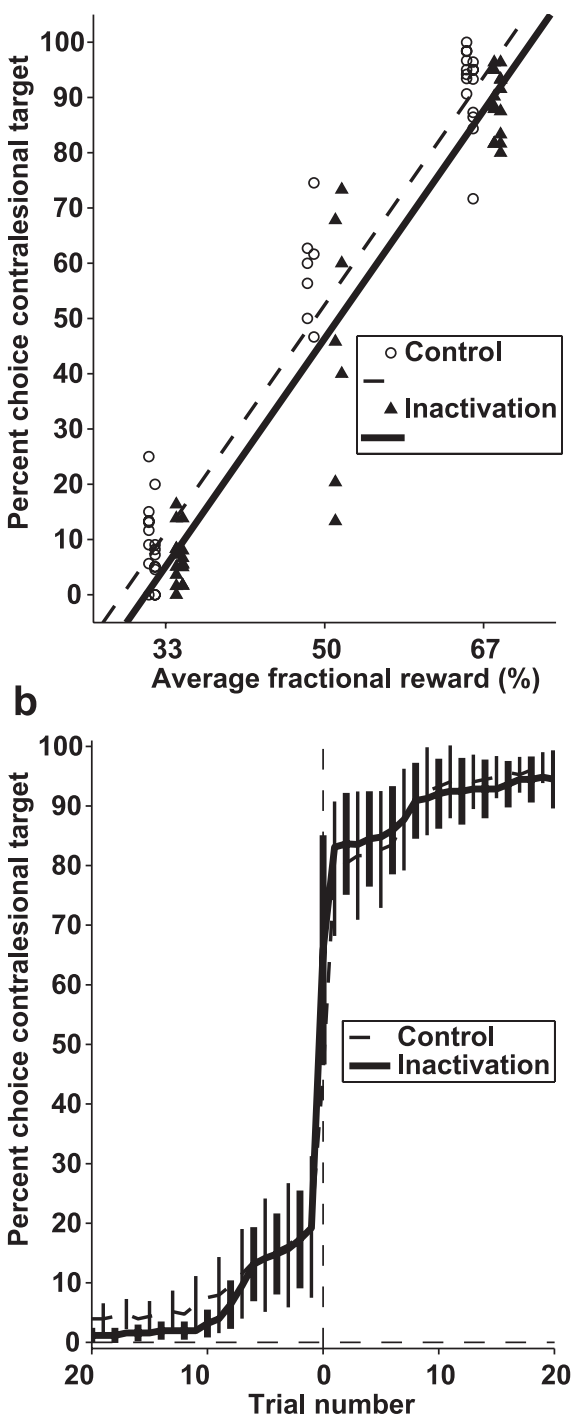

C

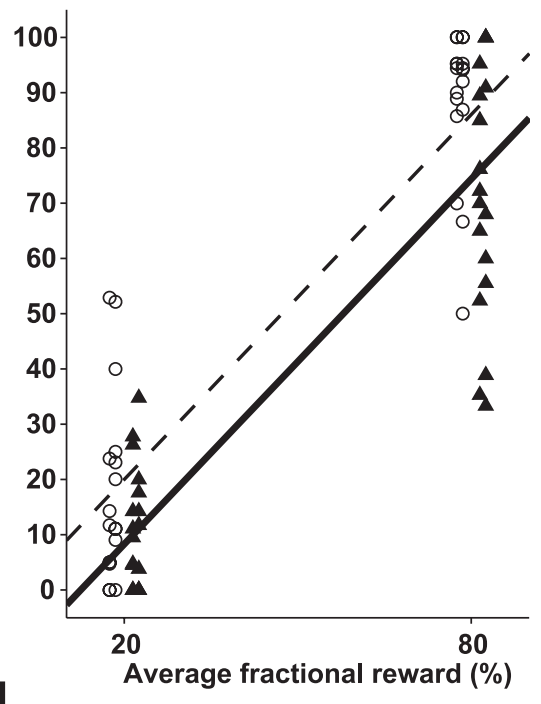

d

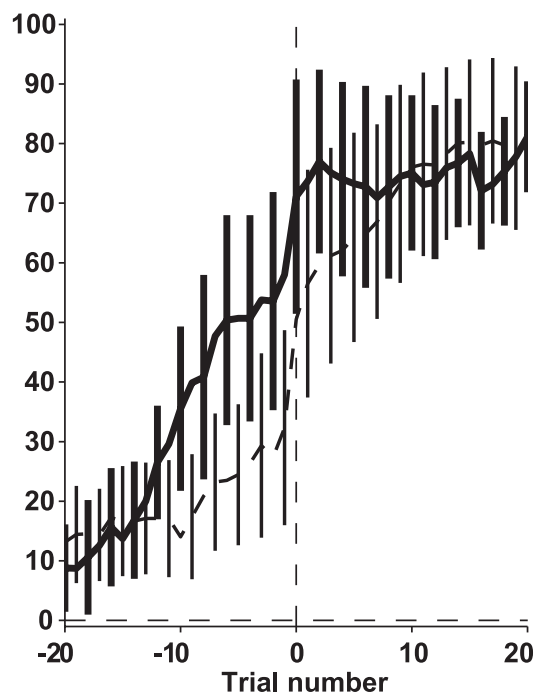

Figure 7. Free-choice saccade tasks. $\boldsymbol{a}$, Percentage contralesional choices as a function of fractional reward in the space-reward task. Each point represents a single session, and lines are simultaneous fits through the data using ANCOVA. For clarity of presentation, points representing control and inactivation sessions were slightly separated along the horizontal axis. $\boldsymbol{b}$, Temporal profile of choice behavior on the space-reward task, in all inactivation (solid) and control (dashed) sessions in which the contralesional target started by having the lower reward value. Trials were aligned on the reversal point (trial 0 ), and the average fraction of choice of the contralesional target was calculated in a sliding window (width 10 trials, stepped 1 trials, bounded at the reversal trial). Error bars represent confidence intervals of the mean across sessions. Bins with significant differences between control and inactivation sessions (one-way ANOVA, $p<0.05$ ) are indicated with an asterisk. c, $\boldsymbol{d}$, Data from the color-reward task in the same format as in $\boldsymbol{a}$ and $\boldsymbol{b}$.

case), and there was no correlation between the magnitude of the spatial and nonspatial effects of the inactivation $(r=0.09, p=$ 0.81 ). Thus, injection volume and the quantity of injected muscimol were not significant factors in our results.

\section{Reward expectation}

Among the more consistent factors modulating LIP activity is reward expectation. Neurons show reward sensitivity in freechoice tasks as well as in instructed tasks that do not involve active decisions and encode both the probability and the magnitude of expected reward (Platt and Glimcher, 1999; Sugrue et al., 2004). This raises the possibility that inactivation may produce specific impairments in decisional processes, i.e., the monkeys' ability to achieve an accurate estimation of expected reward.
To test this possibility, we tested performance on two free-choice tasks in which monkeys had to choose between targets providing different amount of reward.

In the spatial version of the task (six experiments in each monkey) reward magnitude was linked to target location, such that either the contralesional or the ipsilesional target initially received the larger reward and contingencies reversed without warning after 60-70 trials.

To examine spatial and nonspatial deficits, we fitted the fraction of choices of the contralesional target as a function of fractional reward of that target using ANCOVA (Fig. 7a). In this analysis, a spatial bias would be expressed as a change in the intercept, whereas a change in reward sensitivity would produce a change in slope. We found a significant drop in the intercept on inactivation relative to control sessions, indicating a bias away from the contralesional field (intercepts at 0 fractional reward, $-72.9 \%$ for inactivation vs $-67.5 \%$ for control; $p=0.02$ ). However, inactivation and control data yielded identical slopes, indicating no effect on sensitivity to reward per se $(2.4 \%$ choice/percentage fractional reward; $R^{2}=0.84 ; p=0$ for the regression). This result was obtained when including the entire dataset, as well as after excluding four outlier experiments in which the fraction of choices in control sessions was unusually low. The regression results were further confirmed with a two-way ANOVA (entire dataset), which revealed significant effects of fractional reward $(p=0)$ and inactivation $(p<0.02)$ but no interaction between the variables $(p=0.85)$.

We considered several additional ways in which the inactivation may have affected reward decisions. If inactivation disrupted the memory of location-reward associations, this may have caused higher variability in the preference for the highreward target. To evaluate this possibility, we measured the consistency of highreward choices on a session-by-session basis, as the variance in the running average of the fraction of high-reward choices across a session. There was no significant effect of inactivation on choice variance $(104.0 \pm 20.4$ in control sessions vs $126.8 \pm 19.3$ in inactivation sessions; $p=0.28)$. Another possibility is that inactivation may have altered the monkeys' ability to track dynamically changing reward contingencies. However, the temporal profile of choice probability across a session (Fig. 7b) showed that monkeys quickly adjusted their preference after the reversal in reward contingencies with a time course that was indistinguishable in control and inactivation sessions (two-way ANOVA, $p=$ 0 for trial position, $p=0.39$ for inactivation effect, and $p=0.97$ for interaction). Monkeys showed slight anticipation of the reversal point (choice preference begins to change slightly before 
trial 0), which occurred consistently after 60-70 trials. (Note that, although running averages are convolved with a boxcar filter, the size of the filter was adjusted so that data before and after the reversal are never averaged together; the anticipation seen in these figures is therefore real and not attributable to a filtering artifact.) Another possibility is that the effects of the inactivation may have depended on the difficulty of the decision: although no effects may be found when the two targets are associated with clearly different rewards, effects may be revealed if the alternatives are more similar to each other and the decision is more difficult. To examine this possibility, we computed the difference between control and inactivation choices for each fractional reward value. The inactivation effect was independent of fractional reward (changes in contralesional choices were $-3.1 \pm 2.8$, $-2.8 \pm 11.7,-3.8 \pm 10.5$, and $-2.8 \pm 0.9 \%$ for fractional rewards of $26,41,59$, and $74 \% ; p=0.9$, one-way ANOVA). Finally, although the above analyses focused on choice probability, it is possible that inactivation may have prolonged the time needed for a decision. However, although inactivation elevated SRTs for contralesional targets (two-way ANOVA, $p<10^{-4}$ for effect of inactivation on contralesional targets, $p>0.5$ for ipsilesional targets), the effect of inactivation was unrelated to reward ( $p=$ 0.29 for interaction term). In summary, inactivation introduced a constant ipsilesional bias in choice probability but no measurable effects in reward evaluation per se.

Similar results were found on the color-reward task (Fig. $7 c, d)$. This task was similar to the space-reward task, except that one target was red and the other was green (with their respective locations randomized from trial to trial), and reward was linked to target color. As in the space-reward task, the best fit of the data was with two lines of identical slopes $(1.1 \%$ choice/fractional reward) but a significantly lower intercept in inactivation relative to control conditions $(-14$ vs $-2 \% ; p=0.0038)$, showing that inactivation produced a slight ipsilesional bias but did not impair the sensitivity to reward. These results, obtained in the full dataset, were replicated if we excluded three outlier experiments, in which monkeys showed unusually low reward preference in control sessions. As for the space-reward task, there was no significant effect of inactivation on the within-session choice variance $(161 \pm 37.6$ in control vs $131 \pm 31.3$ in inactivation sessions; $p=$ $0.4 ; n=10$ sessions) and no effect on the time course of preference reversal around the reversal trial (Fig. $7 d)$. SRTs were significantly elevated for contralesional $\left(p<10^{-5}\right)$ but not ipsilesional $(p=0.1)$ targets, without a significant interaction between inactivation and reward (both $p>0.15$ ).

We note that, although we used only two clearly distinct values of fractional reward, the color task was more challenging than the spatial task. First, the overall fraction of choices of the highreward target was lower in the color-reward than in the spacereward task $(85.8 \pm 12.6$ vs $91.50 \pm 6.2 ; p<0.05)$. This can be seen in the lower slope of choices as a function of fractional reward (Fig. $7 b$ vs $a$ ) and in the more sluggish dynamics of preference reversal at the reversal trial (Fig. $7 d$ vs $c$ ) in the color relative to the spatial task. Although in the color task monkeys showed an earlier anticipatory tendency than in the space task, this reflected worse performance, because anticipatory choices, especially when starting so long before reversal, lowered accumulated reward. Thus, tracking the value of a variable-location target appeared more difficult than tracking a reward that was fixed to a spatial location.

On the space-reward task, monkeys showed a slight contralesional bias in the control condition (choices were above $50 \%$ at equal fractional reward), but this bias remained constant across experiments, providing no evidence for long-term damage ( $p>$ 0.3 , one-way ANOVA). To evaluate the possibility that our injection volumes may have been insufficient, we estimated reward sensitivity as the difference in fraction of contralesional choices between low- and high-reward conditions and then estimated the inactivation effect as the difference between this sensitivity measure on inactivation and control sessions. The inactivation effect was not correlated with either injection volume or injection amount or with the contralesional spatial bias on either the spaceor the color-reward task (all $p>0.10$ ).

\section{Discussion}

Using reversible inactivation with the GABA agonist muscimol, we tested the hypothesis that LIP is involved in three nonspatial computations reflected in its neural activity: the planning of a nontargeting manual report, the predictive control of saccades based on estimation of elapsed time, and reward-based decisions. However, although inactivation produced reliable visuospatial deficits, it had no measurable effects on nonspatial aspects of performance, suggesting that LIP plays a minor role in these nonspatial computations. We first discuss several methodological considerations that can potentially limit the interpretation of the results and the implications of the findings for the functions of LIP and of the parietal cortex in general.

\section{Methodological and conceptual considerations}

Because our conclusions rely critically on the absence of a nonspatial deficit, it is important to rule out sources of type II errors: confounds that may have caused us to miss a nonspatial deficit if one were truly there. A first possible concern is that our inactivation may not have been sufficiently extensive to produce a deficit. However, to safeguard against this possibility, we used amounts of muscimol that matched or exceeded those used in previous studies: 24-66 $\mu \mathrm{g}$ at each site compared with 8-24 $\mu \mathrm{g}$ in the study by Li et al. (1999) and 12-24 $\mu \mathrm{g}$ in the study by Wardak et al. (2004). Although Wardak et al. (2004) made several separate small injections in each experiment, we opted against this approach because of the potential for inflicting permanent nonspecific mechanical damage. Nevertheless, the visuospatial deficits we obtained were similar in magnitude to those reported by Wardak et al. (2004) and Li et al. (1999), establishing the efficacy of our procedures, and in no case did the behavioral impairment (spatial or nonspatial) correlate with the volume or weight of injected muscimol. Thus, the size and volume of the lesion were not limiting factors in the results.

A second possible concern is that the tasks we used were too easy to reveal a deficit. However, optimal performance clearly required an intact LIP, because inactivation produced significant deficits in each task. Moreover, the target selection components of the task, which were consistently impaired, were arguably easier than the nonspatial components, which were spared. In the temporal anticipation task, the chief source of difficulty was estimating the complex delay distribution across multiple trials, whereas the saccade itself was a simple nonspeeded movement to a clearly visible, isolated target, yet inactivation elevated SRT for contralesional saccades but spared the computation of elapsed time. Similarly, in the reward tasks, the chief challenge was dynamically updating reward contingencies across trials, whereas the saccade itself required only selection among two widely spaced suprathreshold targets, yet it was this target selection, and not the reward evaluation, that was affected by inactivation. Finally, in no case was there a trend for the effects to depend on difficulty within a task. For instance, had difficulty been a significant factor in the temporal anticipation task, we would expect to obtain 
the largest impairments at the lowest anticipation values (intermediate delays), but no such trend was visible in the data (Fig. 6). Similarly, we saw no trend for a deficit to appear in more ambiguous conditions of the space-reward task (50\% fractional reward) or in the more challenging color-reward task. Thus, it was not the difficulty of the task but the nature of the underlying computation that determined the magnitude of the inactivation effect.

A third concern is that some nonspatial effects may have been too subtle to be picked up in our data. To safeguard against this possibility, we performed multiple analyses on each dataset (e.g., analyzing SRTs as a function of delay and anticipation in the temporal hazard task; analyzing the evolution of choices and reaction times in the reward task), we examined both the first moment (mean/median) and second moment (variance) of the distributions, and we performed each analysis both on an experiment-by-experiment basis and at the population level. For nonspatial effects, $p$ values did not even approach significance, whereas spatially specific deficits were readily apparent not only in the aggregate data but also in a large majority of individual experiments.

A fourth concern is related to the nature of spatial or nonspatial coding. It may be argued that, if nonspatial information is more widely distributed throughout cortical and subcortical centers, a lesion in any single area may not produce a significant deficit. However, distributed coding per se cannot explain our results, because the nonspatial functions analyzed here are affected by focal lesions in other areas (Gallese et al., 1994, 1997; Fogassi et al., 2001; Buhusi and Meck, 2005; Fanselow and Poulos, 2005; Ponnusamy et al., 2007). Conversely, visuospatial information is also widely distributed throughout the visual system, and yet it was consistently affected by focal inactivation of LIP.

A related concern is that we might have revealed nonspatial deficits had we made bilateral inactivations of LIP. Although LIP neurons show the strongest nonspatial modulations when the attended stimulus is in their RF (typically in the contralateral hemispace), small effects are also found if attention is directed out of the RF (Oristaglio et al., 2006). However, inactivating the small residual signal in the ipsilesional hemisphere is very unlikely to have a significant effect, given that there was no effect of silencing the bulk of the signal in the contralesional hemisphere. In addition, the results of bilateral inactivation are difficult to interpret, because they would most likely not produce the spatial bias that is a critical control in our experiment.

A sixth and final concern is that performance after inactivation does not indicate the function of the inactivated area but reveals the capacity of the spared brain to compensate for loss of that area. However, although this is a significant concern in the case of permanent lesions, its importance is minimized by our use of transient inactivation. Although our results cannot strictly rule out the possibility that LIP affects nonspatial computations in the intact brain, they set clear bounds on the importance of these contributions. The findings show that any nonspatial function that LIP may have in the intact brain is fully compensated for within minutes of LIP inactivation, but such compensation is not possible for spatial functions. Thus, the findings reveal a true specialization of LIP for spatial over nonspatial processing.

\section{Three nonspatial functions}

Limb motor planning

Although much evidence implicates LIP in oculomotor control, the possibility that it is also important for skeletal movement has not been definitively ruled out. LIP directly abuts, and has reciprocal connections with, parietal areas implicated in limb motor planning, including the anterior intraparietal area, the parietal reach area, and areas 7a and 7b (Lewis and Van Essen, 2000a,b; Nakamura et al., 2001). LIP neurons respond to limb motor planning in a range of task conditions, including visually guided reach movements (Snyder et al., 1997) and nontargeting manual responses (Maimon and Assad, 2006; Oristaglio et al., 2006). Despite these responses, the present results do not support the idea that LIP directly contributes to limb motor planning. The muscimol-induced deficits on the covert search task (Fig. 2), although robust, were captured fully by the hemifield of the target regardless of limb; there was no global deficit in manual performance, nor a specific deficit related to a limb or target-limb configuration.

\section{Temporal anticipation}

Temporal processing is thought to involve a broad distributed network that partly overlaps with areas implicated in space perception (Griffin et al., 2001, 2002; Buhusi and Meck, 2005; Battelli et al., 2008). In monkeys, LIP activity is modulated by elapsed time in tasks in which monkeys explicitly report stimulus duration (Leon and Shadlen, 2003) or prepare a simple visually guided saccade (Janssen and Shadlen, 2005). However, the present results argue that LIP is not critically involved in the computation of elapsed time: LIP inactivation spared the dependence of SRT on delay and anticipation even while it reliably elevated SRTs for saccades to contralesional targets.

We note that, although the results from the anticipation task were negative, the deficits on the double-saccade task (Fig. 1) suggest that LIP may be involved in a different form of time judgment: the perception of the relative timing of rapid visual events. In humans, parietal damage slows the dynamics of attention and vision as measured in attentional blink and apparent motion tasks (Shapiro et al., 2002; Husain and Nachev, 2007; Battelli et al., 2008). However, these deficits are found in both contralesional and ipsilesional fields and at the center of gaze, whereas our data show a specific slowing of target detection in the contralesional field. Thus, it remains to be established to what extent our results indicate a global slowing in visual/attentional dynamics that extends beyond a simple impairment in spatial processing.

\section{Reward expectation}

Among the best documented influences on LIP activity is information about expected reward (Sugrue et al., 2005), and the presence of reward information has been interpreted as indicating a role of LIP in oculomotor decisions (Platt and Glimcher, 1999). However, it has been questioned whether LIP is a primary source of reward evaluation or whether it merely integrates reward information into a spatial priority map (Maunsell, 2004; Sugrue et al., 2004; Gottlieb, 2007). Our results provide direct support for the latter possibility. To the extent that LIP inactivation impaired reward-based decisions, it did so by introducing a spatial bias-a tendency to avoid a contralesional target-without affecting measures of the decision process itself, such as sensitivity to fractional reward, choice variance, or the time needed for a decision. Thus, LIP is necessary for using reward information in spatially unbiased manner but not for evaluating expected reward per se.

Of course, no single experiment can test the involvement of an area in the full gamut of potential nonspatial functions. In particular, LIP neurons also show global changes in activity that encode task context or rules independently of (before) visual stimulation (Stoet and Snyder, 2004; Balan and Gottlieb, 2006), and our experiment does not speak to the significance of these 
global contextual changes. However, the three functions we tested here represent an excellent test-bed for a class of nonspatial responses that are expressed in gated manner in LIP, i.e., as modulations of a primary visuospatial/attentional response. Our results provide direct evidence that these modulations reflect feedback to a visuospatial representation and not involvement of LIP in nonspatial computations.

\section{Relation to the human parietal lobe}

In humans, evidence from functional imaging and neuropsychological testing suggests a functional gradient, whereby superior (dorsal) parietal areas tend to have predominantly spatial visual and motor functions, whereas inferior parietal areas have more pronounced nonspatial functions (Husain and Nachev, 2007). The intraparietal sulcus, which marks the border between superior and inferior areas, shows activations in both spatial and nonspatial tasks. The present results seem to suggest that LIP may be analogous to superior parietal cortex or to the transitional intraparietal area. A firm conclusion in this direction depends on better understanding of homologies between monkey and human parietal cortex and of the human homolog of area LIP.

\section{References}

Balan PF, Gottlieb J (2006) Integration of exogenous input into a dynamic salience map revealed by perturbing attention. J Neurosci 26:9239-9249.

Balan PF, Oristaglio J, Schneider DM, Gottlieb J (2008) Neuronal correlates of the set-size effect in monkey lateral intraparietal area. PLoS Biol 6:e158.

Barash S, Bracewell RM, Fogassi L, Gnadt JW, Andersen RA (1991) Saccaderelated activity in the lateral intraparietal area. I. Temporal properties. J Neurophysiol 66:1095-1108.

Battelli L, Walsh V, Pascual-Leone A, Cavanagh P (2008) The "when" parietal pathway explored by lesion studies. Curr Opin Neurobiol 18:120-126.

Bisley JW, Goldberg ME (2003) Neuronal activity in the lateral intraparietal area and spatial attention. Science 299:81-86.

Buhusi CV, Meck WH (2005) What makes us tick? Functional and neural mechanisms of interval timing. Nat Rev Neurosci 6:755-765.

Dias EC, Segraves MA (1999) Muscimol-induced inactivation of monkey frontal eye field: effects on visually and memory-guided saccades. J Neurophysiol 81:2191-2214.

Dias EC, Kiesau M, Segraves MA (1995) Acute activation and inactivation of macaque frontal eye field with GABA-related drugs. J Neurophysiol 74:2744-2748.

Fanselow MS, Poulos AM (2005) The neuroscience of mammalian associative learning. Annu Rev Psychol 56:207-234.

Fogassi L, Gallese V, Buccino G, Craighero L, Fadiga L, Rizzolatti G (2001) Cortical mechanism for the visual guidance of hand grasping movements in the monkey: a reversible inactivation study. Brain 124:571-586.

Gallese V, Murata A, Kaseda M, Niki N, Sakata H (1994) Deficit of hand preshaping after muscimol injection in monkey parietal cortex. Neuroreport 5:1525-1529.

Gallese V, Fadiga L, Fogassi L, Luppino G, Murata A (1997) A parietofrontal circuit for hand grasping movements in the monkey: evidence from reversible inactivation experiments. In: Parietal lobe contributions to orientation in $3 \mathrm{D}$ space (Thier P, Karnath H-O, eds), pp 619-631. Heidelberg: Springer.

Gottlieb J (2007) From thought to action: the parietal cortex as a bridge between perception, action, and cognition. Neuron 53:9-16.

Gottlieb JP, Kusunoki M, Goldberg ME (1998) The representation of visual salience in monkey parietal cortex. Nature 391:481-484.

Griffin IC, Miniussi C, Nobre AC (2001) Orienting attention in time. Front Biosci 6:D660-D671.
Griffin IC, Miniussi C, Nobre AC (2002) Multiple mechanisms of selective attention: differential modulation of stimulus processing by attention to space or time. Neuropsychologia 40:2325-2340.

Husain M, Nachev P (2007) Space and the parietal cortex. Trends Cogn Sci 11:30-36

Ipata AE, Gee AL, Goldberg ME, Bisley JW (2006) Activity in the lateral intraparietal area predicts the goal and latency of saccades in a freeviewing visual search task. J Neurosci 26:3656-3661.

Janssen P, Shadlen MN (2005) A representation of the hazard rate of elapsed time in macaque area LIP. Nat Neurosci 8:234-241.

Leon MI, Shadlen MN (2003) Representation of time by neurons in the posterior parietal cortex of the macaque. Neuron 38:317-327.

Lewis JW, Van Essen DC (2000a) Mapping of architectonic subdivisions in the macaque monkey, with emphasis on parieto-occipital cortex. J Comp Neurol 428:79-111.

Lewis JW, Van Essen DC (2000b) Corticocortical connections of visual, sensorimotor, and multimodal processing areas in the parietal lobe of the macaque monkey. J Comp Neurol 428:112-137.

Li CS, Mazzoni P, Andersen RA (1999) Effect of reversible inactivation of macaque lateral intraparietal area on visual and memory saccades. J Neurophysiol 81:1827-1838.

Maimon G, Assad JA (2006) A cognitive signal for the proactive timing of action in macaque LIP. Nat Neurosci 9:948-955.

Maunsell JH (2004) Neuronal representations of cognitive state: reward or attention? Trends Cogn Sci 8:261-265.

Mesulam MM (1999) Spatial attention and neglect: parietal, frontal and cingulate contributions to the mental representation and attentional targeting of salient extrapersonal events. Philos Trans R Soc Lond B Biol Sci 354:1325-1346.

Nakamura H, Kuroda T, Wakita M, Kusunoki M, Kato A, Mikami A, Sakata H, Itoh K (2001) From three-dimensional space vision to prehensile hand movements: the lateral intraparietal area links the area V3A and the anterior intraparietal area in macaques. J Neurosci 21:8174-8187.

Oristaglio J, Schneider DM, Balan PF, Gottlieb J (2006) Integration of visuospatial and effector information during symbolically cued limb movements in monkey lateral intraparietal area. J Neurosci 26:8310-8319.

Padoa-Schioppa C, Assad JA (2006) Neurons in the orbitofrontal cortex encode economic value. Nature 441:223-226.

Platt ML, Glimcher PW (1997) Responses of intraparietal neurons to saccadic targets and visual distractors. J Neurophysiol 78:1574-1589.

Platt ML, Glimcher PW (1999) Neural correlates of decision variables in parietal cortex. Nature 400:233-238.

Ponnusamy R, Poulos AM, Fanselow MS (2007) Amygdala-dependent and amygdala-independent pathways for contextual fear conditioning. Neuroscience 147:919-927.

Shapiro K, Hillstrom AP, Husain M (2002) Control of visuotemporal attention by inferior parietal and superior temporal cortex. Curr Biol 12:1320-1325.

Snyder LH, Batista AP, Andersen RA (1997) Coding of intention in the posterior parietal cortex. Nature 386:167-170.

Stoet G, Snyder LH (2004) Single neurons in posterior parietal cortex of monkeys encode cognitive set. Neuron 42:1003-1012.

Sugrue LP, Corrado GS, Newsome WT (2004) Matching behavior and the representation of value in the parietal cortex. Science 304:1782-1787.

Sugrue LP, Corrado GS, Newsome WT (2005) Choosing the greater of two goods: neural currencies for valuation and decision making. Nat Rev Neurosci 6:363-375.

Wardak C, Olivier E, Duhamel JR (2002) Saccadic target selection deficits after lateral intraparietal area inactivation in monkeys. J Neurosci 22:9877-9884.

Wardak C, Olivier E, Duhamel JR (2004) A deficit in covert attention after parietal cortex inactivation in the monkey. Neuron 42:501-508. 